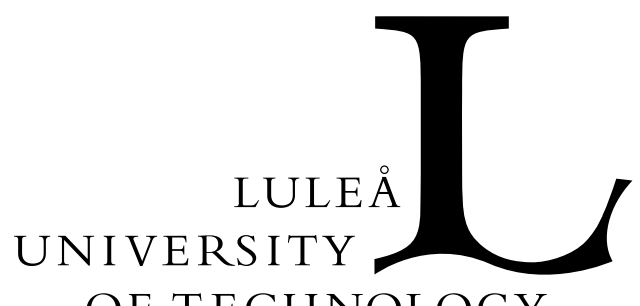

OF TECHNOLOGY

Division of Signal Processing

Visiting address: Universitetsområdet, Porsön, Luleå Postal address: SE-971 87, Luleå, Sweden

Telephone: +46920910 00. Fax: +4692072043

URL: http://www.sm.luth.se/csee/sp/

\title{
Experimental Determination of the Ultrasonic Echo from a Pointlike Reflector Using a Tomographic Approach
}

Anders Grennberg and Magnus Sandell

In Proceedings of IEEE Ultrasonic

Symposium, vol. 2, pp. 639-642, Tucson, USA, October 1992.

(C) 1992 IEEE. Personal use of this material is permitted. However, permission to reprint/republish this material for advertising or promotional purposes or for creating new collective works for resale or redistribution to servers or lists, or to reuse any copyrighted component of this work in other works must be obtained from the IEEE. 


\title{
Experimental determination of the ultrasonic echo from a pointlike reflector using a tomographic approach
}

\author{
Anders Grennberg Magnus Sandell \\ Division of Signal Processing \\ University of Luleå \\ S-951 87 Luleå, SWEDEN
}

\begin{abstract}
One important parameter for an ultrasonic transducer is the echo it yields from a pointlike reflector. It can be measured directly but there are however certain problems connected with this. This reflector must have such a size that it can be considered a point, which results in a very weak echo and consequently a low SNR. We propose an alternative method to calculate the echo from a pointlike reflector by measuring the echoes from sliding halfplanes. Using a tomographic way of reasoning, we can calculate the single point echo from these measurements. Our approach was to find basis functions that are suitable for the inversion of the measurements. These basis functions depend on which norm and weight function you choose. Numerical solutions are given for chosen weight functions and the inversion is shown for the measurements.
\end{abstract}

\section{Introduction}

A very common way of imaging with ultrasonics is to use the pulse-echo method [7]. This method measures the distance to an object by estimating time-of-flight, i.e. the time between transmission of an ultrasonic pulse and the arrival of the echo. However, there is more information in the echoes besides the arrival time, such as the slope of the surface at the measured point. In our experiments we have used a focused airborne ultrasonic transducer with a radius of curvature of 45 $\mathrm{mm}$ and a center frequency of $1.1 \mathrm{MHz}$. Two echoes from a plane surface with a sloping angle of $0^{\circ}$ and $5^{\circ}$ respectively are shown in Fig. 1. The shape of the echo is dependent on the angle of inclination [2], as we can see. The focal point has the same distance from the plane in both measurements.

To be able to extract this information, a detailed knowledge of the transducer is required. Since a complicated object may be considered to be an aggregation

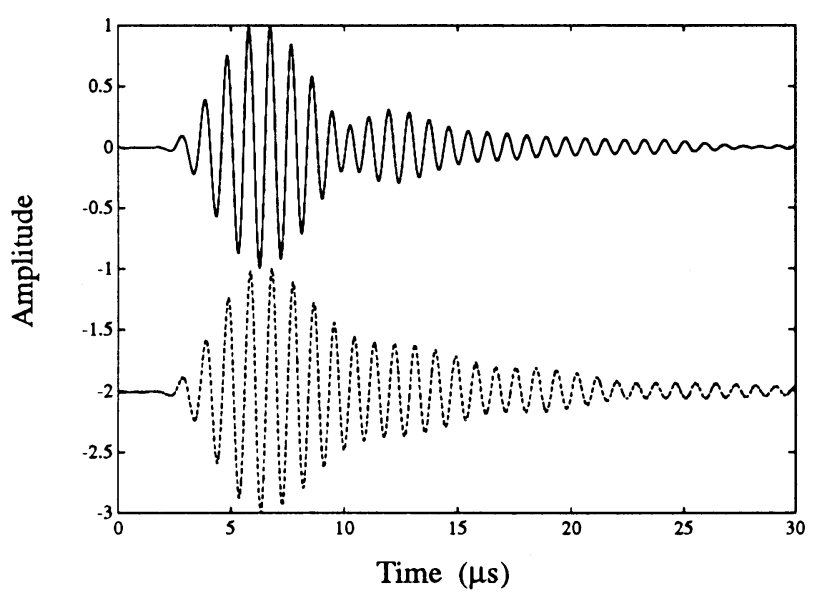

Figure 1: Echoes from plane surfaces with normalized amplitude . Perpendicular (above) and $5^{\circ}$ angle of inclination (below)

of point objects [5], the echo from a pointlike reflector would be of great importance. There are however practical difficulties to measure it directly. The object would have to be very small to be considered pointlike and this would result in a weak echo with low SNR. There is also the effect of diffraction to be considered. We tried instead a tomographic approach. In tomography, the line-integral of a two-dimensional function is normally measured. Methods for inverting these measurement to the original function are known, in a certain extent. The equivalence in our problem would be to measure the echo from an infinitesimal thin line with infinite extent, see Fig. 2. Instead we chose to measure the echoes from halfplanes, i.e. the integrals of the line echoes. The reason for this is a better SNR and a reduction of the diffraction effect originating from echoes from the edges of the object. The physical width of the line will then not be a problem. 


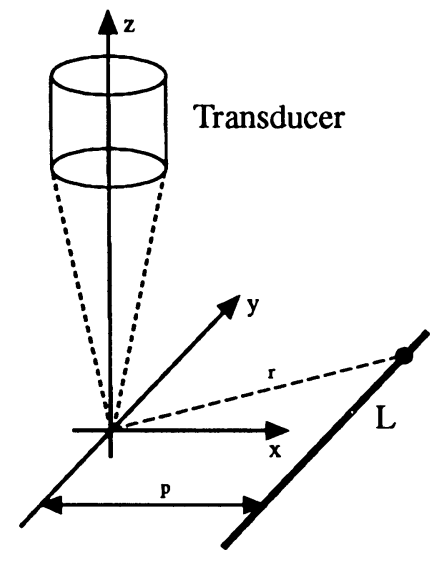

Figure 2: Reflecting line

\section{Theory}

\section{A Formulation of the problem}

In our experiments we have assumed an axisymmetric focused transducer. The reason for this is simplicity, but the method can be extended to unsymmetric transducers. Suppose that we have a line which lies in a plane parallel to the focalplane and is located at a height $z$ from the transducer. The line has a perpendicular distance $p$ to the axis of the transducer, see Fig. 2. The transducer works both as a transmitter and a receiver. The echo $f_{\lambda}$ from the line will be a function of distance $p$, height $z$ and, of course, time $t$. Since the height $z$ was constant in our experiments and $t$ is only a parameter in our calculations, we will drop them from now on.

Using superposition [5], we can express the echo from an infinitesimal thin line with infinite extent in terms of the single point echoes. Let $f(r)$ denote the echo from a single point at a distance $r$ from the axis of symmetry. The echo $f_{\lambda}(p)$ from a line with perpendicular distance $p$ to the axis of symmetry, will then be a line integral of $f(r)$.

$$
\begin{gathered}
f_{\lambda}(p)=\int_{L} f(r) d r= \\
2 \int_{p}^{\infty} f(r) \frac{r}{\sqrt{r^{2}-p^{2}}} d r
\end{gathered}
$$

Since the transducer is axisymmetric, we can assume the line to be parallel to the $y$-axis for the sake of simplicity. The expression in (1) is known as the Abel transform. The inversion of (1) can be found in [1] and is calculated in the following manner:

$$
f(r)=-\frac{1}{\pi} \int_{r}^{\infty} \frac{f_{\lambda}^{\prime}(p)}{\sqrt{p^{2}-r^{2}}} d p
$$

However, there are practical problems in measuring the echo from a line. An object has to be very narrow to be considered a line, which will yield weak echoes with a low SNR. We will also get diffraction effects from the edges. These problems can be solved if we instead measure the echoes from halfplanes. If we denote the echo from a perpendicular halfplane with $f_{\pi}(p)$, where $p$ is the same distance as before (i.e. $f_{\pi}(-\infty)$ is a reflection from complete plane and $f_{\pi}(\infty)$ from an empty space), we get the following relationship between the line echo and the halfplane echo

$$
\begin{gathered}
f_{\pi}(p)=\int_{p}^{\infty} f_{\lambda}(q) d q \\
f_{\pi}^{\prime}(p)=-f_{\lambda}(p)
\end{gathered}
$$

Inserting (3) in (2), we obtain the final expression

$$
f(r)=\frac{1}{\pi} \int_{r}^{\infty} \frac{f_{\pi}^{\prime \prime}(p)}{\sqrt{p^{2}-r^{2}}} d p
$$

\section{B Decomposition of $f_{\pi}(p)$}

The integral (4) can be evaluated numerically. However, since the integrand is singular at its lower bound and we have measurement noise, we cannot evaluate the integral using ordinary quadrature formulae. One way of dealing with this is to use a regularization technique $[3,4]$. Another method would be to borrow an idea from tomography. First we approximate the reflecting target to be a circle or a circlesegment. This means that we will neglect all echoes from points further away from the axis of symmetry than a certain distance. Furthermore we will normalize this distance to be $r=1$. This results in the approximations $f(r)=0$ for $|r|>1$ and consequently $f_{\pi}(p)=0$ for $p>1$. If $f(r)$ is the single point echo, we measure $f_{\pi}(p)=(S f)(p)$ where $S$ is the integral operator

$$
f_{\pi}(p)=(S f)(p)=\int_{A_{p}} f(r) d A
$$

As before, $p$ denotes the perpendicular distance between the edge of the halfplane and the axis of symmetry. The reflecting area of the target $A_{p}$ is the integration area and $d A$ an area element. These quantities are depicted in Fig. 3. To catch essential features of the functions involved we introduce the weighted Hilbert spaces

$$
\begin{array}{r}
\mathcal{H}=L^{2}\left([0,1], W_{H}(r)\right) \\
\mathcal{K}=L^{2}\left([-1,1], W_{K}(p)\right)
\end{array}
$$

By doing a singular value decomposition of the operator $S[6]$, we can find two orthonormal bases $\left\{\phi_{i}(r)\right\}$ 


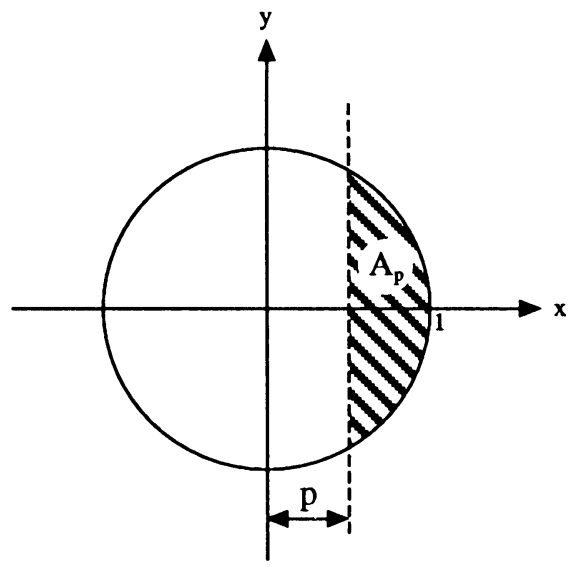

Figure 3: Variables used in calculations

and $\left\{\gamma_{i}(p)\right\}$ for the Hilbert spaces $\mathcal{H}$ and $\mathcal{K}$ respectively. The relation between the two basis are

$$
\left(S \phi_{i}\right)(p)=\sigma_{i} \gamma_{i}(p) \quad i=1,2,3 \ldots
$$

where $\sigma_{i}$ are the singular values of $S$. Decomposing the halfplane echoes $f_{\pi}(p)$ into $N$ basis functions results in an approximation

$$
\hat{f}_{\pi}(p)=\sum_{i=1}^{N} c_{i} \gamma_{i}(p)
$$

The error consists of modeling errors, truncation error and measurement noise. The latter is not in $\mathcal{K}$. We therefore project the measurements to the subspace spanned by the functions $\gamma_{i}(p), i=1,2 \ldots N$ using the standard inner product and norm. This means that we will do a least square fitting to obtain the coefficients $c_{i}, i=1,2 \ldots N$. Using (8) and (9), we have an approximation of $f(r)$ as

$$
\hat{f}(r)=\sum_{i=1}^{N} c_{i} \sigma_{i}^{-1} \phi_{i}(r)
$$

\section{Choosing proper weight functions}

In Fig. 4, typical shapes of $f_{\pi}(p)$ are shown for different values of the time $t$. This appearance is quite natural since $f_{\pi}(-\infty)$ is a reflection from a complete plane and $f_{\pi}(\infty)$ is an echo from an empty space. This information should be used in the inversion process. By choosing a weight function $W_{K}(p)$ that becomes very large as $p \rightarrow 1$, we will force the basis functions $\left\{\gamma_{i}(p)\right\}$ to be small in that area. A natural assumption for $f(r)$ is that $f(r)=O\left((1-r)^{\alpha}\right)$ as $r \rightarrow 1$ with $\alpha \geq 1$. From (5) we can deduce that $(S f)(p)=O\left((1-p)^{\frac{3}{2}+\alpha}\right)$ as $p \rightarrow 1$. With these observations we can define the weight functions $W_{H}(r)$ and $W_{K}(p)$ in the form $(1-r)^{\beta}$ and $(1-p)^{\gamma}$ respectively, with such exponents $\beta$ and $\gamma$ that $f \in \mathcal{H}$ implies $S f \in \mathcal{K}$.

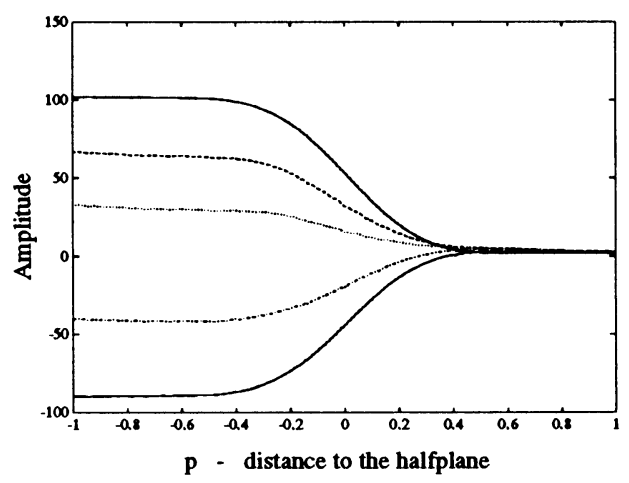

Figure 4: Typical shapes of $f_{\pi}(p)$

\section{Experiments}

We have done our experiments on a perspex block using a focused airbourne transducer. It had a radius of curvature $R=45 \mathrm{~mm}$, a cross-section radius $a=10 \mathrm{~mm}$ and a center frequency of $1.1 \mathrm{MHz}$. In order to measure $f_{\pi}(p)$ we placed the transducer just above the edge of the perspex block. We moved the transducer over the edge using a coordinatetable and for each position we measured the echo 1000 times. An average of these echoes was then stored in a computer. The sample frequency was $20 \mathrm{MHz}$ and we took 1024 samples which makes the sweep length $51.2 \mu \mathrm{s}$. This time is enough for the echo to vanish completely. Between each measurement we moved the transducer $\Delta p=12.5 \mu \mathrm{m}$. The perspex block was placed perpendicular to the transducer's movement. After the measurements, we found that the echo's amplitude was in the order of the noise's when the perspex block was further away than $2 \mathrm{~mm}$ from the axis of symmetry. This is the distance that we normalize to $p=1$ in our calculations.

\section{Results}

For the chosen weight functions we have calculated the approximations of the corresponding basis functions for $S$. This has been done by computing a weighted SVD. The first three basis functions for $\mathcal{H}\left(\right.$ i.e $\phi_{i}$ ) and $\mathcal{K}\left(\right.$ i.e. $\gamma_{i}$ ) are shown in Fig. 5 and 6 respectively.

Using these basis functions we have inverted the halfplane integrals $f_{\pi}(p)$ and obtained the single point echoes. Since $f_{\pi}(p)$ depends on the time $t$ we will have 


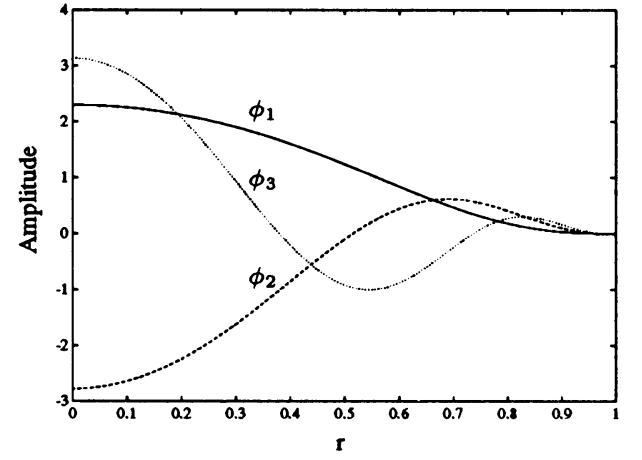

Figure 5: The first three basis functions $\phi_{i}$ for $\mathcal{H}$

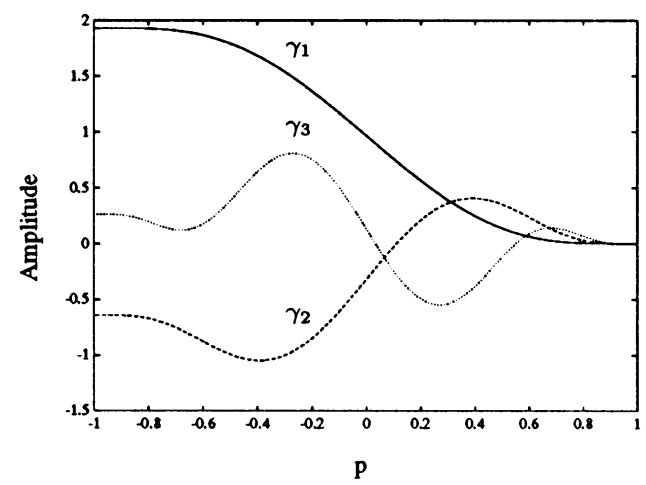

Figure 6: The first three basis functions $\gamma_{i}$ for $\mathcal{K}$

one inversion for every sampled point in time. Including the time dependence, we have constructed the echo from a pointlike reflector $f(r, t)$. One echo of special interest is that from a point at the focal point. This it plotted in Fig. 7.

\section{Summary}

In this article we have presented a method to extract an essential parameter for ultrasonic transducers, namely the single point echo (SPE). It is possible to measure the SPE directly by using a point reflector. Due to a low SNR and diffraction effects we have chosen another method. By using ideas from tomography, we measure integrals of the desired SPE instead. In tomography, the line integral would be considered, i.e. the echo from an infinitesimal thin line. We have measured the halfplane integrals instead to improve the SNR. To find the SPE, these measurements can be inverted by decomposing the halfplane echoes in suitable basis functions. These were found by introducing Hilbert spaces with weight functions to use the a priori knowledge of the signals. The basis function were calculated numerically

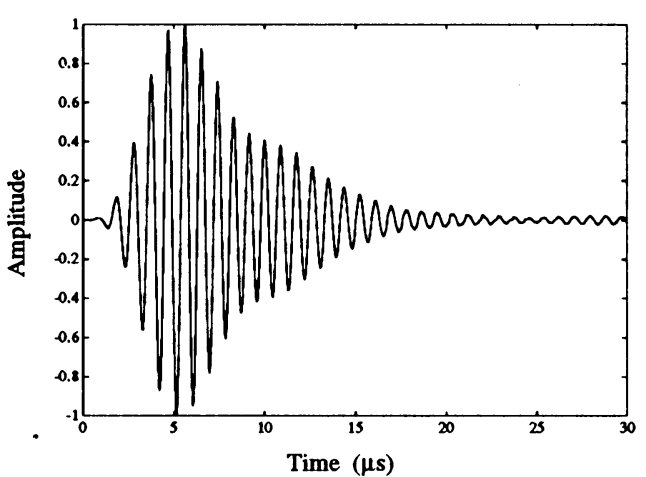

Figure 7: The calculated echo from a point reflector at the focal point

and the SPE's were found by a truncated singular value decomposition.

\section{References}

[1] Bracewell, R.N.; The Fourier transform and its applications, 2nd ed., McGraw-Hill, 1986.

[2] Börjesson, Per Ola; Holmer, Nils-Gunnar; Lindström, Kjell \& Salomonsson, Göran; "Notes on ultrasound echoes from angular surfaces", Technical report TR-172, Electrical Engineering Department, University of Lund, September 1982.

[3] Grennberg, Anders \& Sandell, Magnus; "Experimental determination of the single point echo of an ultrasonic transducer using a tomographic approach", in 14th Annual International Conference of the IEEE Engineering in Medicine and Biology Society, (forthcoming)

[4] Herman, Gabor T. \& Naparstek, Abraham; "Fast image reconstruction based on a Radon inversion formula appropriate for rapidly collected data", Siam J. Appl.Math., vol. 33, no. 3, pp. 511-533, November 1977.

[5] Lhémery, Alain; "Impulse-response method to predict echo-responses from targets of complex geometry. Part 1", J. Acoust. Soc. Am., vol 90, no 5, pp 2799-807, 1991.

[6] Natterer, F.; "The mathematics of computerized tomography", Wiley, 1986.

[7] Sundström, N.; Börjesson, P.O.; Holmer, N.-G.; Olsson, L. \& Persson, H.W.; "Registration of surface structures using airborne focused ultrasound", Ultrasound in medicine and biology, vol. 17 , no. 5, 1991. 